\title{
Demo: Multi-user virtual world simulator of F-16 aircraft engine mechanical maintenance
}

\author{
Paulo Fernandes, André Pinheiro, Gonçalo Cruz, Ana \\ Margarida Maia \\ ECT - Escola de Ciências e Tecnologia, Dep. Engenharias \\ UTAD - University of Trás-os-Montes e Alto Douro \\ Vila Real, Portugal \\ \{pklides, andrepinheiro.infor\}@gmail.com, \\ \{goncaloc,margaridam\}@utad.pt,
}

\author{
Leonel Morgado, Paulo Martins, Hugo Paredes, \\ Benjamim Fonseca \\ INESC TEC (formerly INESC Porto) \\ UTAD - University of Trás-os-Montes e Alto Douro \\ Vila Real, Portugal \\ \{leonelm, pmartins, hparedes, benjaf\}@utad.pt
}

\author{
J. Bernardino Lopes, José Cravino \\ CIDTFF - Centro de Investigação em Didática e Tecnologia na Formação de Formadores \\ UTAD - University of Trás-os-Montes e Alto Douro \\ Vila Real, Portugal \\ \{blopes, jcravino\}@utad.pt
}

\begin{abstract}
This demonstration proposal aims to present a 3D multi-user training simulator, developed in the 3D OpenSimulator virtual world platform, in order to enable the practice and training of mechanical maintenance for F-16 engines, specifically the installation of a Pratt \& Whitney F100 engine in a F-16 aircraft - a process that requires three skilled engine technicians to do a set of cooperative execution tasks. The main purpose of the simulator is to provide trainees and trainers with more opportunities to conduct training, enabling technician training to be enhanced with cooperation and context prior to the training phase with actual physical engines. In this proposal, we describe the architecture of the system and the sample simulation tasks.
\end{abstract}

Keywords - 3D Simulators; virtual worlds; OpenSimulator; training; aircraft engine maintenance

\section{INTRODUCTION}

The training of technical procedures for maintenance of specific engines is a common and frequent process at Portuguese Air Force. It has an initial theory phase, based on technical documents known as "Technical Orders" or TOs [1]. Then, an on-the-job training phase ensues, with trainees acting directly on an engine, in actual maintenance circumstances.

This final on-the-job training phase is resource-demanding, since it requires engines to be available for training, and consequently not to be available for service. Also, procedure errors in training may, in some cases, produce costly component damage. Further, several of the technical procedures need to be executed by a team, meaning that time allocation of different trainees, trainers, and experienced technicians need to be managed, in order for a full team to be available for on-the-job training to ensue. These various resource requirements place constraints on the availability of on-the-job training opportunities and emphasize the need to optimize it. The development of a 3D multi-user mechanical training simulator for this scenario thus aims to allow trainees to reach on-the-job training better prepared and thus to optimize the effectiveness of the resource-intensive training occasions with physical engines. This is a joint effort of the Portuguese Air Force, the University of Trás-os-Montes e Alto Douro (UTAD), and INESC TEC.

\section{SYSTEM ARCHITECTURE}

From a software engineering perspective, there is also a goal of lessening the resource requirements of simulation development, by conducting it in a readily available virtual world platform but implementing the control code and decision-making logic at an external system. The rationale for this architectural choice was to focus the software development effort on the simulation behavior in a way that could be rendered independent from the graphical platform of user interaction [2].
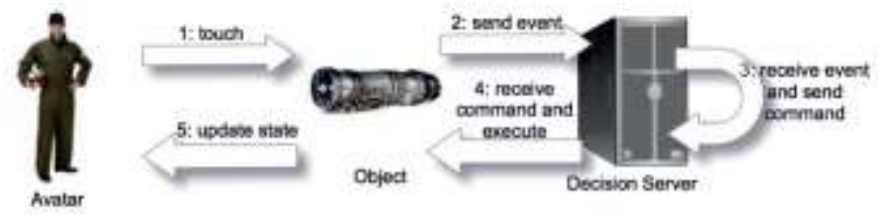

Fig. 1. System architecture (adapted from [3])

We took advantage of the fact that OpenSimulator virtual world can execute scripts concurrently, with each script able to communicate with external software servers, to develop the architecture presented in figure 1. Therefore, whenever the avatar of a technician interacts with the system (phase 1 in the figure), its actions can be detected by the scripts normally, but instead of having the actions hard coded into the scripts, the scripts report them to an external system (phase 2) for overall state control and decision-making (phase 3). This system can 
also check for cooperation and synchronization requirements (i.e., tasks that need to be performed in concert by several technicians). The server then provides the script with an answer to its report (phase 4), and the script reacts, causing the visual appearance of the simulation to change, and thus providing the technicians' avatars with a new state for their intervention (phase 5). We emphasize that the 3D environment does not conduct any decision-making: it is only responsible for reporting to the web service the events triggered by the technician's avatars or other objects and then wait for commands issued by the Web Service, in response to these reports. In this sense, it is an embryonic implementation of the Model-View-Controller architectural style [4] for virtual worlds. This holds the potential to render decision-making independent from the 3D environment, not just for this system but as a generic approach.

\section{SAMPLE SIMULATOR TASKS}

The installation process of a Pratt \& Whitney F100 engine in an F-16 aircraft is quite extensive and complex, requiring three technicians to do the various procedures. Plus, a specific role in the process is that of process checker, which may lead to a fourth person being involved, should none of the three required technicians have the credentials to perform this role.

All necessary procedures are specified in a document known as the Job Guide, which refers to all the Technical Orders containing the necessary information. Installation of the engine inside the aircraft fuselage, the first procedure being implemented in the prototype, is a single process, but it is typically subdivided into four jobs, known as PT1, PT2, PT3, and PT4. Currently the prototype is implemented to support simulation throughout the PT1 job, which is a precondition for executing jobs PT2, PT3, and PT4. But more important, it enables testing of the cooperation situations of the process, because this PT1 job requires the involvement of all technicians, as it entails several tasks that cannot be performed by a single individual.

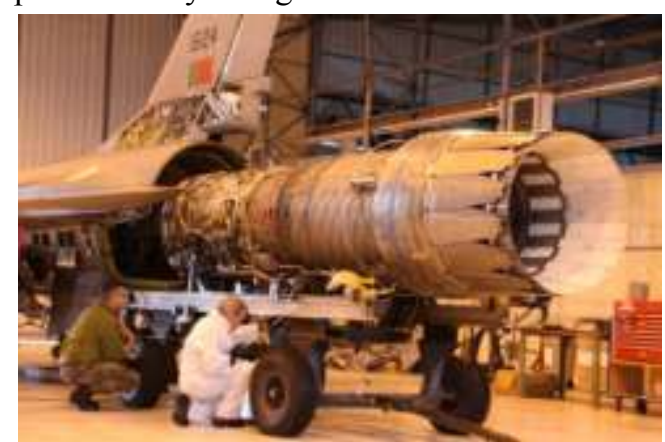

Fig. 2. Mechanics raising the engine at Air Base

One such task is the raising of the engine to align it with the empty hull of the aircraft fuselage, which requires all 3 technicians: two on the left side, another on the right side, as shown in figure 2. When the process checker issues the command (i.e., this may be the fourth element in the team), the three technicians will operate screw driving machines in concert to lift the motor simultaneously and coordinately, using speech as a means of synchronization, to avoid tilting the engine excessively while raising it.

The multi-user virtual world platform OpenSimulator enables three or four trainees to practice the synchronization in this task in a similar way, as long as voice chatting is available. In the current prototype, once all preconditions are adequate (such as having steadied the bearing cart and having adequate tools in hand and fitting for the tools in place), the engine lifting can take place. Once it is initiated, we elected to implement it in the following way: the simulator will take control of the arrow keys and change their function. During the lifting process, up and down arrow keys will respond as if operating the direction of screw driving machines or the turning of lifting wrenches (figure 3a). The three technicians need to press their respective keys in the correct direction at roughly the same time (using voice chatting to synchronize their actions, as in the physical world) in order to lift or lower the engine. We defined "roughly" as a 2-second interval.

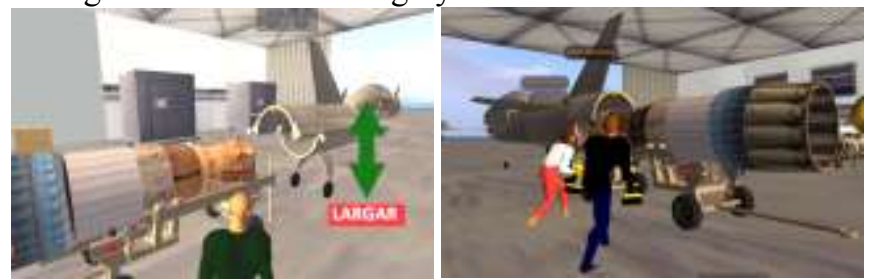

Fig. 3. Simulator aspects: (a) Arrow in virtual space; (b) Mechanics lifting the engine in virtual space

Currently, this is a precondition for lifting/lowering to occur, but in the future we intend to expand the simulator behavior to cover error conditions and accidents caused by wrong operations. Figure $3 \mathrm{~b}$ shows the avatars of trainees during the lifting of the engine in the virtual space. We propose to make available at the conference an OpenSimulator server and three client stations, so that participants can not only witness the simulation environment by experience it and the tasks supported by the simulation.

\section{REFERENCES}

[1] Lockheed-Martin-Corp., TO 1F-16AM-2-70JG-10-21 - Organizational Maintenance - Engine Removal and Installation - Model F100-PW220/220E - USAF/EPAF Series - F-16/B Mid-Life Update aircraft, Technical Manual Job Guide. Bethesda, MD, USA: Lockheed Martin Corporation, 2009.

[2] B. Fonseca, H. Paredes, J. Rafael, L. Morgado, and P. Martins, 'A Software Architecture for Collaborative Training in Virtual Worlds: F16 airplane engine maintenance', in Collaboration and Technology: 17th International Conference, CRIWG 2011, Proceedings, pp. 102-109, Berlin: Springer, 2011.

[3] P. Petridis, I. Dunwell, D. Panzoli, S. Arnab, A. Protopsaltis, M. Hendrix, S. Freitas, Game Engines Selection Framework for HighFidelity Serious Applications, International Journal of Interactive Worlds vol. 2012, pp. 1-19, 2012.

[4] G. Krasner and S. Pope, A cookbook for using the model-view controller user interface paradigm in Smalltalk-80, Journal of ObjectOriented Programming, 1 (3), 26-49, 1988. 